\title{
Neurosarcoidosis, Could It Be? - Case Report
}

\section{Jonathan T. Grossman $^{1 *} \mid$ Katherine Reano ${ }^{2}$ | Alona Kondramashin ${ }^{1} \mid$ Asia Filatov $^{1}$}

*Correspondence: Jonathan T. Grossman

Address: ${ }^{1}$ The Florida Atlantic University Charles E. Schmidt College of Medicine Neurology Residency Program; Boca Raton, Florida; ${ }^{2}$ The Florida Atlantic University Charles E. Schmidt College of Medicine Internal Medicine Residency Program; Boca Raton, Florida

e-mail $\bowtie$ : jgross25@health.fau.edu

Received: 27 July 2021; Accepted: 09 August 2021

Copyright: (C) 2021 Grossman JT. This is an open-access article distributed under the terms of the Creative Commons Attribution License, which permits unrestricted use, distribution, and reproduction in any medium, provided that the original work is properly cited.

\section{ABSTRACT}

While the clinical presentation of systemic sarcoidosis is well established, identification of isolated neurologic sarcoidosis remains a diagnostic challenge. Isolated neurologic sarcoidosis, or neurosarcoidosis, presents in a manner that is often indistinguishable from other neurological diseases. Common clinical features include headache, encephalopathy, gait disturbance, seizure, behavioral abnormalities, sleep difficulties, loss of appetite, cranial mononeuropathy (including visual, auditory, and vestibular dysfunction), myelopathy, radiculopathy, myopathy, and peripheral neuropathy. While probable diagnosis requires only evidence of inflammation on cerebrospinal fluid studies or magnetic resonance imaging, tissue biopsy with pathologic findings of noncaseating granulomas is required for definitive diagnosis. We present the case of a 54-year-old male with headache, episodic confusion, and gait disturbance attributed to probable neurologic sarcoidosis.

Keywords: Neurologic Sarcoidosis, Neurosarcoidosis, Angiotensin-Converting Enzyme, Sarcoidosis

\section{Introduction}

Sarcoidosis is characterized by a multi-system inflammatory response with a variety of clinical manifestations. Neurosarcoidosis is arguably one of the most unpredictable and heterogenous of these manifestations (Ibitoye et al., 2016). One study found that just under 50\% of its participants ultimately diagnosed with sarcoidosis presented with a neurologic complaint as the initial manifestation of disease (Stern et al., 1985). Neurological symptoms are estimated to occur in just 5-10\% of patients diagnosed with sarcoidosis and both the peripheral and central nervous system can be affected (Voortman et al., 2019). Common clinical features include headache, encephalopathy, gait disturbance, seizure, behavioral abnormalities, sleep difficulties, loss of appetite, cranial mononeuropathy (including visual, auditory, and vestibular dysfunction), myelopathy, radiculopathy, myopathy, and peripheral neuropathy (Finsterer and Grisold, 2015). Prompt diagnosis of isolated neurologic sarcoidosis is often difficult due to the nuanced presentation of this disease and the lack of specificity of diagnostic testing. The aim of this case report is to highlight the importance of a multi-disciplinary approach in expeditious and accurate diagnosis of neurologic sarcoidosis which is often indistinguishable from other neurological diseases. 


\section{Case Presentation}

A 54-year-old black male with past medical history of polysubstance use disorder including cocaine and alcohol presented to our emergency department for evaluation of recurrent, episodic confusion. The first episode occurred approximately 24 hours prior to arrival while he was driving; he recalled the entire event but described the abrupt onset of unfamiliarity with his surroundings causing him to miss the turn to his destination. He then became nauseous and vomited. His wife was in the car at the time and corroborated this account saying that the entire episode occurred over the span of a few minutes. This confusion occurred again shortly following arrival to the hospital, again lasting for a few minutes but this time with associated blurred vision in the bilateral periphery. The only other symptoms he reported were decreased appetite and weight loss.

He denied having any chronic medical conditions or prior surgeries. His wife described him as having a history of "heavy alcohol and cocaine use" that he had reportedly discontinued altogether 4 months prior.

Vitals were entirely unremarkable upon arrival. Remarkable labs were as follows: erythrocyte sedimentation rate (ESR) $26 \mathrm{~mm} / \mathrm{hr}$ (normal range 0-14), sodium $128 \mathrm{mmol} / \mathrm{L}(\mathrm{N}=138-148$ ), chloride $93(\mathrm{~N}=100-108)$, hemoglobin A1c 6.0\% ( $\mathrm{N}=<5.7)$, folate $11.8 \mathrm{ng} / \mathrm{mL}(\mathrm{N}=2.7-17)$, vitamin B12 929 $\mathrm{pg} / \mathrm{mL}(\mathrm{N}=211-911)$, C-reactive protein (CRP) $<0.29 \mathrm{mg} / \mathrm{dL}(\mathrm{N}=<0.80)$, total cholesterol $170 \mathrm{mg} / \mathrm{dL}$ (normal high < 200), LDL 116 (normal high < 129), TSH 1.150 mU/L (N 0.45-4.5 mU/L), urine osmolality $558 \mathrm{mOsm} / \mathrm{kg}$ (N 500-850), urine sodium $70 \mathrm{mEq} / \mathrm{L}$, negative urine drug screen, and serum ethanol level $<3 \mathrm{md} / \mathrm{dL}$ (normal high < 10). Electrocardiogram showed normal rate, rhythm, and axis with right bundle branch block; no prior was available for comparison. Preliminary imaging including nonenhanced CT head and chest radiograph were unremarkable.

A comprehensive neurological exam was performed by our Neurology service in the emergency department. He was alert and oriented to person, place, time, and situation. He described in detail the events leading up to his arrival. The remainder of the exam was otherwise normal with the exception of gaze evoked nystagmus. He did describe feeling a sort of mental "fogginess" over the past month which he thought may be getting worse.

He was placed on Clinical Institute Withdrawal Assessment Alcohol Scale Revised (CIWA-AR) protocol and admitted for further evaluation. Additional labs including a liver function panel and rapid plasma reagin (RPR) were unremarkable. EEG and MRI brain were performed to rule out epileptiform activity and structural abnormalities, both of which were unrevealing. 
Upon re-evaluation on the second day of his stay, he complained of neck pain, occipital headache, and clumsy gait. These symptoms in concert with the above MRI findings prompted concern for potential demyelinating disease vs. meningoencephalitis. Lumbar puncture (LP) was performed after which he was started on empiric antibiotics (ampicillin, ceftriaxone, vancomycin) and acyclovir (Hersi et al., 2021). MRI brain with contrast was ordered revealed a linear pattern of leptomeningeal enhancement within the posterior cranial fossa and anterior aspect of the right sylvian fissure on T1 post-contrast imaging (axial view in Figure 1 and coronal view in Figure 2).

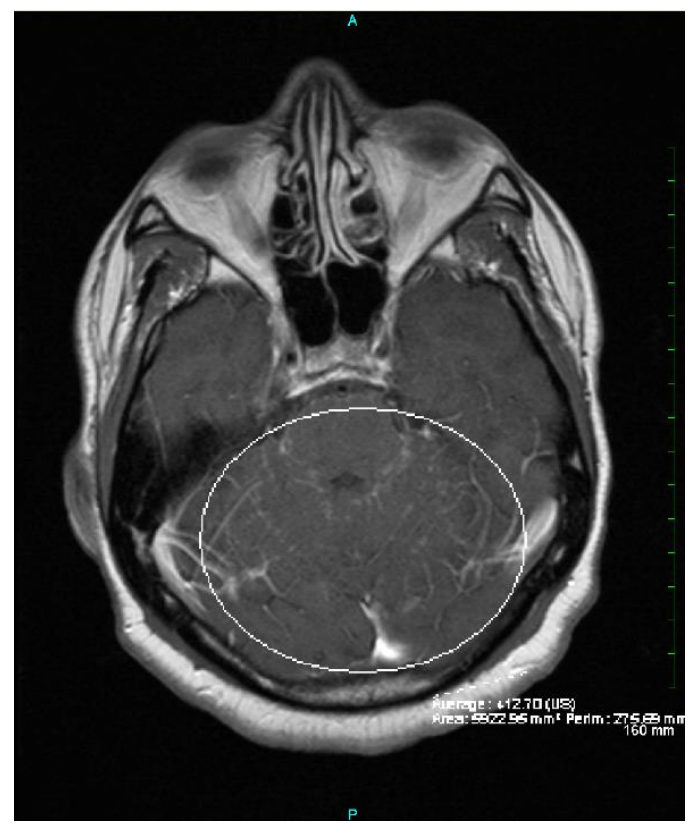

Figure 1: Axial T1-weighted image showing linear pattern of leptomeningeal enhancement within the posterior cranial fossa (see circle for area of interest).

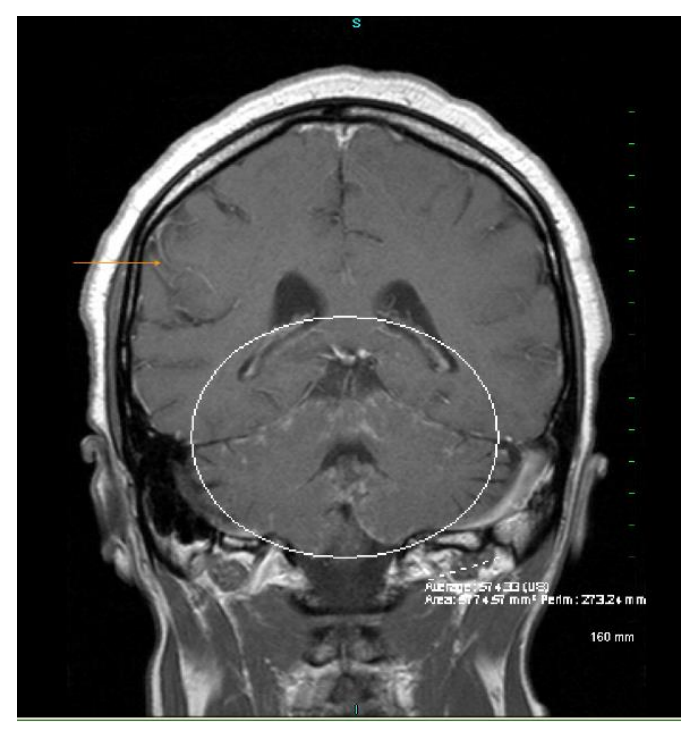

Figure 2: Coronal T1 post-contrast image showing patchy enhancement of the tentorium cerebelli with apparent enhancement in right sylvian fissure (see circle for area of interest). 
Cerebrospinal fluid (CSF) was clear and negative for xanthochromia but was remarkable for pleocytosis 127 (N 0-4) with mononuclear predominance, hypoglycorrhachia $33 \mathrm{mg} / \mathrm{dL}$ (N 40-75), and elevated protein $191 \mathrm{mg} / \mathrm{dL}$ (N 15-45) suggestive of aseptic meningitis.

At this point, we began to consider neurosarcoidosis as a potential diagnosis. A paraneoplastic panel and serum and CSF angiotensin-converting enzyme (ACE) levels were ordered. CT chest with contrast was without hilar adenopathy or parenchymal involvement characteristic of systemic sarcoidosis. CT abdomen and pelvis with contrast was unrevealing for occult malignancy.

In spite of ongoing antimicrobial regimen, he began to experience fevers with persistence of head, neck, and back pain. MRI spine with contrast showed enhancement along the descending cauda equina nerve roots (Figure 3).

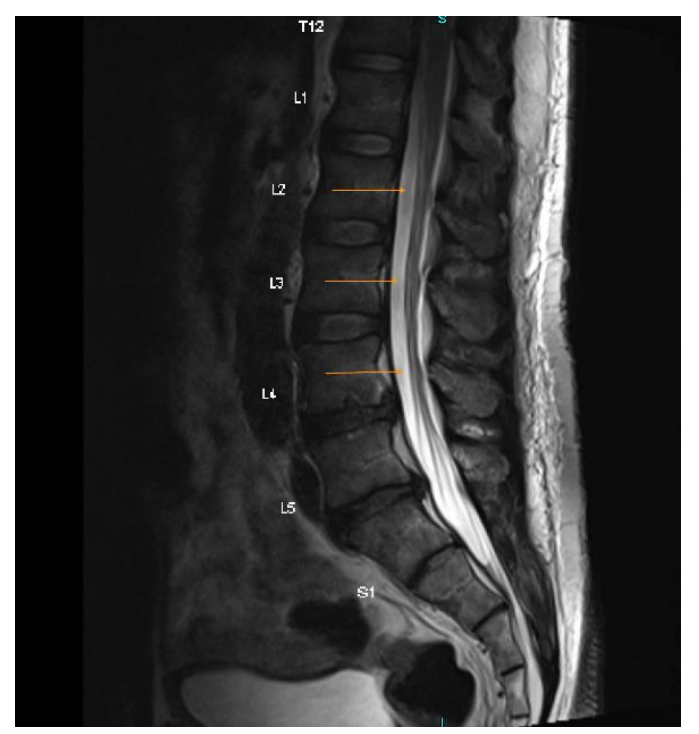

Figure 3: Sagittal T1 post-contrast image with enhancement of descending cauda equina nerve roots.

His fevers resolved soon after and serum and CSF cultures remained without growth; antibiotics were eventually discontinued. In spite of negative serum and CSF herpes simplex virus (HSV) and varicella zoster virus (VZV), IV acyclovir was continued at the discretion of our Infectious Disease specialist with plan to complete a 14-day course (Watson et al., 2017). He was started on a trial of methylprednisolone by our team after which both his headache and mental fog began to improve. Pending CSF studies eventually showed presence of oligoclonal bands 5 (N 0-1), elevated IgG index 1.10 ratio (N 0.28-0.66) and elevated CSF ACE 6.0 unit/L (N 0.0-2.5). He was discharged with plan for reassessment at our clinic following completion of a 2-week steroid course. Unfortunately, he was lost to follow-up.

He returned to our hospital almost 1 year later with recurrence of a similar constellation of 
symptoms at which time MRI was repeated and showed leptomeningeal enhancement with similar distribution to prior studies. He was evaluated again by our inpatient Neurology and Neuro-oncology services who agreed regarding the probable diagnosis of neurologic sarcoidosis.

\section{Discussion}

Neurologic sarcoidosis, or neurosarcoidosis, refers to the neurologic manifestations of the systemic inflammatory response that characterizes sarcoidosis (Lacomis, 2011). Sarcoidosis is known to be most common in those of Swedish and African-American descent (Brodin and Davis, 2017). Isolated neurological sarcoidosis i.e. without systemic involvement is thought to be rare. All tissues of the nervous system are known to be affected. The pathophysiology underlying the symptoms of neurosarcoidosis is thought to be due to inflammatory mediated destruction of myelin with nerve and muscle biopsy specimens remarkable for epineural granulomas and endoneurial infiltrates (Paglia, 2019). Common manifestations include cranial neuropathies such as unilateral 7th nerve palsy or facial diplegia, encephalopathy, aseptic meningitis, myopathy, myelopathy, radiculopathy, peripheral neuropathy, and neuroendocrine dysfunction (Keane, 1994).

Our patient presented with a constellation of symptoms including headache, neck and back pain, confusion, transient visual changes, gait difficulties, and reduced appetite with weight loss.

His headache, neck, and back pain could be explained by leptomeningeal involvement in the form of aseptic meningitis. There is no validated means of distinguishing aseptic meningitis related to neurosarcoidosis from that observed in viral infections, HIV, TB, or syphilis. The recurrent episodes of confusion are most consistent with encephalopathy which can be difficult to distinguish from a multitude of other CNS diseases that may present with encephalopathy including but not limited to syphilis and primary angiitis of the CNS. His transient blurred vision and gait imbalance could be explained by cranial neuropathies affecting visual and vestibular function.

The hypothalamus is involved in approximately 5\% of cases of sarcoidosis (Ropper et al., 2014a). Symptoms can include polyuria as well as alterations in behavior, sleep patterns, appetite, and basal body temperature (Eckel-Mahan and Sassone-Corsi, 2013). Although often accompanied by facial nerve palsy and hilar lymphadenopathy, neuroendocrine dysfunction can be the primary manifestation of disease. Infundibuloneurohypophysitis due to infiltration of the neurohypophysis and pituitary stalk by lymphocytes and plasma cells is postulated to be one mechanism underlying such a global hypothalamic syndrome (Abe, 2008). Neuroendocrine dysfunction can be considered a reliable explanation for his reduced appetite and approximately 20-lb. weight loss as well as his disrupted sleep patterns and 
behavioral changes (e.g. apathy) reported by his wife. He also experienced intermittent fevers of uncertain origin during his hospitalization which could have been occurring as a consequence of hypothalamic inflammation.

While sarcoid neuropathies are infrequently reported, they do occur in the form of polyneuropathy, polyradiculopathy, or mononeuropathies. These neuropathies may be associated with other signs of CNS involvement including diabetes insipidus or myelopathy (Dahan et al., 2016). Our patient did complain of pain/stiffness in the neck and back however there was no evidence of cord compromise on imaging so these symptoms were more likely attributable to radiculopathy or meningeal inflammation. One often unrecognized manifestation is a small-fiber neuropathy characterized by impaired perception of pain and temperature in the distal extremities.

Aside from the well characterized forms of neuropathy, sarcoid myopathy has been described as slowly progressive often painless proximal or distal muscle weakness with elevated creatine kinase level and muscle biopsy showing numerous non-caseating granulomas (Chawla, 2011). Effective treatment of neurological symptoms of sarcoidosis has been seen with moderate doses of corticosteroids (prednisone, 25-50mg daily) (Ropper et al, 2014b). While it appeared that our patient's gait difficulties were of vestibular nature, he did report lower extremity weakness which was not appreciated upon objective exam.

Unfortunately, the clinical manifestations of isolated neurologic sarcoidosis are often indistinguishable from other neurological diseases. Further, there are no definitive pathognomonic findings on neurodiagnostic testing. The most commonly employed diagnostic tests include magnetic resonance imaging and lumbar puncture after which a probable diagnosis can be made. A definitive diagnosis requires biopsy with findings of noncaseating granulomas. As for any investigation of underlying CNS disease, a contrast study is necessary to investigate for meningeal or parenchymal enhancement caused by disruption of the blood-brain barrier in the setting of any active inflammatory state. Both parenchymal and meningeal masses as well as hydrocephalus have been observed in patients with neurosarcoidosis. Our patient had evidence of leptomeningeal enhancement in the posterior cranial fossa, right sylvian fissure, and cauda equina (Roy et al., 2010).

CSF abnormalities are also common including mononuclear pleocytosis, elevated protein, hypoglycorrhachia, elevated IgG index, presence of oligoclonal bands and elevated CSF ACE concentration. Patients with active disease tend to have higher cell counts and total protein (Wengert et al., 2013). Our patient's CSF was remarkable for all of these findings. However, a reliable reference range for CSF ACE concentration remains to be established. While these values can be elevated in infection, 
carcinomatous meningitis, as well as in cases of increased levels of serum ACE, our patient had no identifiable source of infection, his paraneoplastic panel was unremarkable, and serum ACE was not elevated.

While there has yet to be a large scale, randomized controlled trial exploring treatment endpoints for neurosarcoidosis there is general consensus that these patients should first be treated with glucocorticoids. The appropriate dosage and duration are typically dependent upon the site of neurologic involvement. Had he followed up for re-assessment and was found to be without improvement in his symptoms, immunomodulatory therapies (e.g. methotrexate, azathioprine, mycophenolate mofetil) have shown efficacy in treating some patients with refractory symptoms (Agbogu., 1995). Consideration of the potential benefits and adverse effects associated with glucocorticoid versus immunomodulatory therapy should be weighed under the supervision of a neurologist.

\section{Conclusion}

Isolated neurologic sarcoidosis, or neurosarcoidosis, is considered rare and remains a diagnostic challenge due to the wide variability of clinical presentation with symptoms that overlap with other neurological diseases. This case report highlights the importance of a multidisciplinary approach in ensuring expeditious diagnosis. The treatment for isolated neurologic sarcoidosis parallels that of systemic sarcoidosis and our patient experienced interval improvement in his symptoms following initiation of IV methylprednisolone.

\section{References}

Abe T. Lymphocytic infundibulo-neurohypophysitis and infundibulo-panhypophysitis regarded as lymphocytic hypophysitis variant. Brain Tumor Pathol 2008; 25: 59-66.

Agbogu BN, Stern BJ, Sewell C, Yang G. Therapeutic considerations in patients with refractory neurosarcoidosis. Arch Neurol 1995; 52: 875-879.

Brodin P and Davis MM. Human immune system variation. Nat Rev Immunol 2017; 17: 21-29.

Chawla J. Stepwise approach to myopathy in systemic disease. Front Neurol 2011; 2:49.

Dahan A, Brines M, Niesters M, Cerami A, van Velzen M. Targeting the innate repair receptor to treat neuropathy. Pain Rep 2016; 1 : e566.

Eckel-Mahan K and Sassone-Corsi P. Metabolism and the circadian clock converge. Physiol Rev 2013; 93: $107-135$.

Finsterer J and Grisold W. Disorders of the lower cranial nerves. J Neurosci Rural Pract 2015; 6: 377-391.

Hersi K, Gonzalez FJ, Kondamudi NP. Meningitis. [Updated 2021 Feb 26]. In: StatPearls [Internet]. Treasure Island (FL): StatPearls Publishing; 2021 Jan-. Available from: https://www.ncbi.nlm.nih.gov/books/NBK459360/ 
Ibitoye RT, Wilkins A, Scolding NJ. Neurosarcoidosis: A clinical approach to diagnosis and management. J Neurol 2017; 264: 1023-1028.

Keane JR. Bilateral seventh nerve palsy: analysis of 43 cases and review of the literature. Neurology 1994; 44: 1198-1202.

Lacomis D. Neurosarcoidosis. Curr Neuropharmaco 2011; 9: 429-436.

Paglia F, D’Angelo L, Armocida D, Sampirisi L, Giangaspero F, De Vincentiis L, Santoro A. A rare case of spinal epidural sarcoidosis: case report and review of the literature. Acta Neurol Belg 2021; 121: 415-420.

Ropper AH, et al. Diseases of Muscle in Adams and Victor's Principles of Neurology. Eleventh edition. New York: McGrawHill, 2014b. Print.

Ropper AH, et al. The Hypothalamus and Neuroendocrine Disorders in Adams and Victor's Principles of Neurology. Eleventh edition. New York: McGraw-Hill, 2014a. Print.

Roy K, Tripathy P, Senapati A, Saha SK. Intradural Extramedullary Sarcoidosis case report and review of literature. Asian J Neurosurg 2010; 5: 87-90.

Stern BJ, Krumholz A, Johns C, Scott P, Nissim J. Sarcoidosis and its neurological manifestations. Arch Neurol 1985; 42: 909-917.

Voortman M, Drent M, Baughman RP. Management of neurosarcoidosis: a clinical challenge. Curr Opin Neurol 2019; 32: 475-483.

Watson WA, Rhodes NJ, Echenique IA, Angarone MP, Scheetz MH. Resolution of acyclovir-associated neurotoxicity with the aid of improved clearance estimates using a Bayesian approach: A case report and review of the literature. J Clin Pharm Ther $2017 ; 42: 350-355$.

Wengert O, Rothenfusser-Korber E, Vollrath B, Bohner G, Scheibe F, Otto C, Hofmann J, Angstwurm K, Ruprecht K. Neurosarcoidosis: correlation of cerebrospinal fluid findings with diffuse leptomeningeal gadolinium enhancement on MRI and clinical disease activity. J Neurol Sci 2013; 335: 124-130. 